\title{
Influences of the state of ionospheric background on ionospheric heating effects
}

\author{
Hao Shuji ${ }^{1}$, Li Qingliang ${ }^{2}$, Che haiqin ${ }^{2}$, Yang Jutao ${ }^{2}$, Yan Yubo ${ }^{2}$, Wu Zhensen ${ }^{1,{ }^{\star}}$, Xu Zhenwen ${ }^{2}$ \\ ${ }^{1}$ Xidian University, Department of Physics, Xi'an, China \\ ${ }^{2}$ China Research Institute of Radiowave Propagation, Qingdao, China
}

\author{
Article history \\ Received February 21, 2013; accepted June 11, 2013. \\ Subject classification: \\ Ionospheric heating, Ionospheric background, Heating effects, Heating parameters, Electron density gradient.
}

\begin{abstract}
According to the well-performed ionospheric heating experiments at Arecibo in the low latitudes as well as at Tromsø in the high latitudes, the large-scale modification effects are simulated under an assumption of equivalent conditions, i.e., with the same effective radiative power and the same ratio of the heating frequency $f_{H F}$ to the critical frequency of ionospheric $\mathrm{F}$ region foF2. The findings are extensively exploited to verify the validation of our model by comparison to the experimental results. Further, a detailed study is carried out on the influences of the background electron density gradient as well as the ratio of $f_{\mathrm{HF}}$ to foF 2 on heating effects. Conclusions are drawn as follows: under certain conditions, a smaller electron density gradient of background ionospheric F region leads to a better ionospheric heating effect; during over-dense heating, the heating effects are enhanced if the ratio of $f_{H F}$ to foF 2 increases, which is slightly limited by the resultant elevation of the reflection height. However, there might be a better ratio range with small values of the ratio of $f_{H F}$ to foF2, e.g., $[0.5,0.7]$ in the current study. Finally, we analyzed how to select heating parameters efficiently under adverse conditions so to obtain relatively effective results.
\end{abstract}

\section{Introduction}

As an active ionospheric research, modification of the ionosphere by powerful high frequency (HF) waves is to date of great scientific interest and rapidly developed in the last several decades since they provide an effective tool for investigations of the Earth environment as well as for understanding various plasma phenomena. The heating experiments were successfully performed firstly at Platteville, Colorado [Utlaut 1970], almost simultaneously at Arecibo [Gordon and Showen et al. 1971], in the early 1970s, and subsequently at other ionospheric heating facilities such as Tromsø, SURA, HAARP (The High Frequency Active Auroral Research Program) and SPEAR (Space Plasma Exploration by Active Radar). A wide variety of fascinating phenomena are observed, some of which are associated with the development of parametric instabilities and ionospheric turbulence, including enhancement of electron temperature [Mantas et al. 1981], generation of plasma oscillations and field-aligned irregularities [Rietveld et al. 2003, Tereshchenko et al. 2006, Wright and Dhillon 2009], excitation of artificial radio and optical emission [Frolov et al. 1999, Pedersen and Carlson 2001, Kosch et al. 2007]. Among the main phenomena, renewed attention is paid to the wave-plasma interaction process leading to nonlinear density perturbations at different scales. Large-scale (kilometer size) irregularities are best interpreted in terms of thermally driven self-focusing of the incident HF electromagnetic wave [Duncan and Sheerin 1985]. Small-scale (meter size) field-aligned striations are generally explained as differential plasma heating from enhanced Langmuir wave collisional dissipation or HF scatter from the induced plasma oscillations [Blagoveshchenskaya et al. 1995]. Concerning the nature and origin of Langmuir wave, a popular view is the theory of thermal parametric (resonant) instability developed at the upper hybrid resonance altitude [Das and Fejer 1979]. Only the O-mode propagating in the vertical or nearly vertical direction reaches the resonance region, and the X-mode is always reflected from the region lying below the resonance one. In the previous heating experiments, field-aligned irregularities, electron density and anomalous absorption all occurred only during O-mode heating [Robinson et al. 1998]. Recently experimental evidence of the excitation of strong small-scale fieldaligned irregularities was presented due to an X-mode HF pump wave radiated in the direction of magnetic zenith [Blagoveshchenskaya et al. 2011].

In most of studies, the HF-induced electron density profile modification is observed only by a few percent. Xu et al. [2009] presented evidence of a density 
depletion by $4-10 \%$ with the heating facility at Tromsø with an effective radiated power $P_{\text {erf }}=90 \mathrm{MW}$ and heating frequency $f_{\mathrm{HF}}=4.04 \mathrm{MHz}$. Isham et al. [1987] reported a decrease of electron density by $3-5 \%$ using the heating facility at Arecibo with $P_{\text {erf }}=100 \mathrm{MW}$ and $f_{\mathrm{HF}}=5.1 \mathrm{MHz}$. Duncan et al. [1988] first reported direct observations of large electron-density depletions of about $30 \%$ with $P_{\text {erf }}=60 \mathrm{MW}$ and $f_{\mathrm{HF}}=3.175 \mathrm{MHz}$. Such large depletions were observed reproducibly only after local midnight in the winter ionosphere for ionospheric densities critical only for the lowest pump frequency available at Arecibo. The state of the background ionosphere is found to be an important factor influencing the observed effects. The resultant heating effects and their magnitudes may be different due to discrepancies of ionospheric background and heating parameter settings in specific research processes.

Therefore, researchers undertook a series of theoretical investigations by simulation of the factors influencing the strength of ionospheric heating effects. The basic theory of ionospheric heating was systematically discussed by Gurevich [1978] who used a set of continuity equation, momentum equation and energy equation. Shoucri et al. [1984] considered the dependences of heating effects on $P_{\text {erf }}$ and $f_{\mathrm{HF}}$, respectively. Hansen et al. [1992] modeled the large-scale HF-induced ionospheric modifications reported by Duncan et al. [1988]. Mingaleva and Mingalev [1997] and Mingaleva et al. [2008] investigated the range of values for $f_{\mathrm{HF}}$ to get the best heating effects in mid- or low-latitude regions, it is $f_{\mathrm{HF}} \approx 0.8 \sim 0.86 f \mathrm{foF} 2$ during the day, and $f_{\mathrm{HF}} \approx 0.9 \sim 0.96 f \circ \mathrm{OF} 2$ at night.

In order to perform a detailed study on diversities of heating effects in different areas, an investigation is carried out on the influences of the background electron density gradient as well as the ratio of $f_{\mathrm{HF}}$ to $f \circ \mathrm{F} 2$ on heating effects in Section 2. Then our final conclusions and proposals for future work are presented in Section 3. A theoretical model of ionospheric heating will be built in Appendix A.

\section{Analysis of simulation results}

\subsection{Comparion of simulations with experimental results}

In order to verify the numerical model described in Appendix A, simulation parameters are selected to be the same with those in the heating experiments listed in Table 1. Density depletions and temperature enhancements are found at the reflection height for both cases at Arecibo as well as at Tromsø. In our simulation, the density depletions $N_{1} / N_{0}$ are about $3.4 \%$ at Arecibo. The maximum temperature in the depletions increases about $28.4 \%$ which is consistent with the experimental results at $20-30 \%$. For the case at Tromsø, we found the density depletion up to $6.12 \%$ and the temperature enhancement reaching $70.6 \%$ which also are in accordance with the measured enhancement of $60-120 \%$.

\subsection{Analysis of factors influencing heating effects}

In the F-region, the heating effects have a close relationship with energy absorption via various electron heating channels. Besides the linear Ohmic heating process, the various nonlinear processes with different thresholds, characteristic times, and saturation mechanisms may also be excited, depending on the incident power and the state of background ionosphere [Duncan et al. 1988]. Thus, with the values of the pump frequency and $P_{\text {erf }}$ selected, the geomagnetic dip and the state of ionospheric background (mainly described by the electron densities and their gradients) have a remarkable impact on heating effects (variation of electron density), which will be dealt with in Section 2.2 separately.

2.2.1. Influences of $P_{\text {erf }}$ and the dip angle on heating effects

Influences of system's $P_{\text {erf }}$ and the geomagnetic dip on heating effects have already been specified in the previous calculations, which will be briefly discussed here. We hence compare the results of heating experiments performed at Tromsø and Arecibo (see Table 1) and find that the two experiments were carried out under differ-

\begin{tabular}{|c|c|c|c|c|c|c|c|}
\hline site & $\begin{array}{l}\text { geographic } \\
\text { coordinate }\end{array}$ & 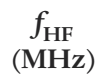 & $\begin{array}{c}\text { foF2 } \\
(\mathrm{MHz})\end{array}$ & $f_{\mathrm{HF}} / f \circ \mathrm{F} 2$ & $\begin{array}{c}h_{0} \\
(\mathrm{~km})\end{array}$ & $T_{\mathrm{e} 1} / T_{\mathrm{e} 0}$ at $h_{0}$ & $N_{1} / N_{0}$ at $h_{0}$ \\
\hline Arecibo & $68.8^{\circ} \mathrm{W}, 18.3^{\circ} \mathrm{N}$ & 5.1 & 7.44 & 0.69 & 200 & $20 \% \sim 30 \%$ & $3 \% \sim 5 \%$ \\
\hline Tromsø & $19.2^{\circ} \mathrm{E}, 69.6^{\circ} \mathrm{N}$ & 4.04 & 4.99 & 0.81 & 217 & $60 \% \sim 120 \%$ & $4 \% \sim 12 \%$ \\
\hline
\end{tabular}

Table 1. Parameters of the heating experiments carried out at Arecibo [Isham et al. 1987] and Tromsø [Xu et al. 2009], where $h_{0}$ refers to the reflection height of HF radio wave in the ionosphere. 


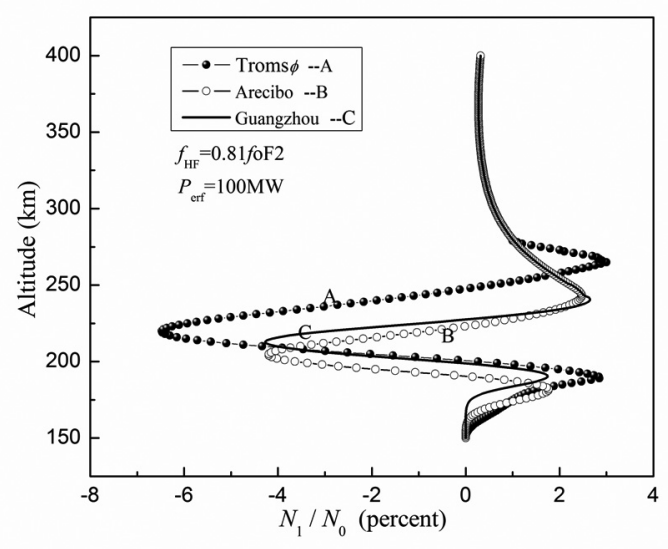

Figure 1. Comparisons of relative variation profiles of electron density at Arecibo, Tromsø and Guangzhou under equivalent conditions with $P_{\text {erf }}=100 \mathrm{MW}$ and $f_{\mathrm{HF}} / f_{\mathrm{OF}} 2=0.81$ indicated in the insert.

ent heating conditions (e.g., different $P_{\text {erf }}$ and $f_{\mathrm{HF}} / f \mathrm{oF} 2$ ), leading to different heating effects. In order to study the influence of $P_{\text {erf }}$ and $f_{\mathrm{HF}} / f \mathrm{OF} 2$ on the heating effects and compare the difference of the heating effects under the same conditions at different radar sites, we theoretically simulate the heating effects assuming the same heating conditions (i.e., $P_{\text {erf }}=100 \mathrm{MW}$ and $f_{\mathrm{HF}} / f \mathrm{oF} 2=0.81$ ) at Tromsø, Arecibo, and Guangzhou. Under the equivalent conditions with $P_{\text {erf }}=100 \mathrm{MW}$ and $f_{\mathrm{HF}} / f \mathrm{oF} 2=0.81$ during daytime, heating effects are simulated for the cases at Tromsø, Arecibo, and Guangzhou $\left(113^{\circ} \mathrm{E}\right.$, $22^{\circ} \mathrm{N}$ ), respectively. The background parameters derived from the IRI and MSIS model at Tromsø and Arecibo are the same as those mentioned in Section 2.1. The relative variation profiles of electron density are plotted in Figure 1. Heating effects are found to be quite comparable between Arecibo and Guangzhou, with density depletions of $4.20 \%$ and $4.27 \%$, respectively. However, the effects are appreciably stronger at Tromsø with a density depletion of $6.47 \%$.

Comparison between the simulations above in Section 2.1 and the results shown in Figure 1 indicates that when the reflection altitude $h_{0}$ remains invariable, the increase of $f_{\mathrm{HF}} / f \mathrm{OF} 2=0.81$ or $P_{\text {erf }}$ of the heating system leads to an enhancement of heating effects. For example, with $f_{\mathrm{HF}} / f_{\mathrm{OF}} 2$ increased from 0.69 to 0.81 at Arecibo, the density depletion ranges from $3.4 \%$ to $4.2 \%$; with the $P_{\text {erf }}$ enhanced from $90 \mathrm{MW}$ to $100 \mathrm{MW}$ at Tromsø, the depth of electron density depletions increases slightly from $6.12 \%$ to $6.47 \%$.

It must be pointed out that the numerical comparisons of the density modification at different locations have limited guiding significance. The regional difference between high- and mid-latitudes and the time difference at similar latitudes are not taken into account. The ionospheric heating experiments were easily carried out after sunset at Arecibo but mainly done during daytime at Tromsø for lack of high enough electron density during night [Rietveld et al. 2002]. At Arecibo, the large depletions were observed reproducibly even after local midnight in the winter ionosphere.

\subsubsection{Influences of the electron density gradients on} heating effects

In order to study the impact of electron density gradients on the density modification in ionospheric heating experiments, we select different background density profiles deduced from two ionograms illustrated in Figure 2 at different times of a day and keep the other parameters fixed in our simulation. The density profiles are labeled as density mode A and B (both derived from Guangzhou ionospheric observatory of CRIRP; Ionogram A and B were deduced at 15:31 LT in October 5, 2010, and 16:13 LT in October 17, 2010, respectively), respectively, as shown in Figure 3. The relative variations of electron density with height caused by heating are shown Figure 4.

The reflection height of HF radio wave $h_{0}$ is about $243 \mathrm{~km}$ for the selected density models shown in Figure 2. From Figure 3. We can see $\nabla N_{A}<\nabla N_{B}$ for the case of $h>h_{0}$ and $\nabla N_{A}>\nabla N_{B}$ for $h<h_{0}$. As illustrated in Figure 4 , a larger density gradient leads to a smaller density depletion and temperature increment, which implies that the density gradient is an adverse factor for ionospheric heating experiments.

The interpretation can be more easily understood in terms of electron density scale height $H$ [White and

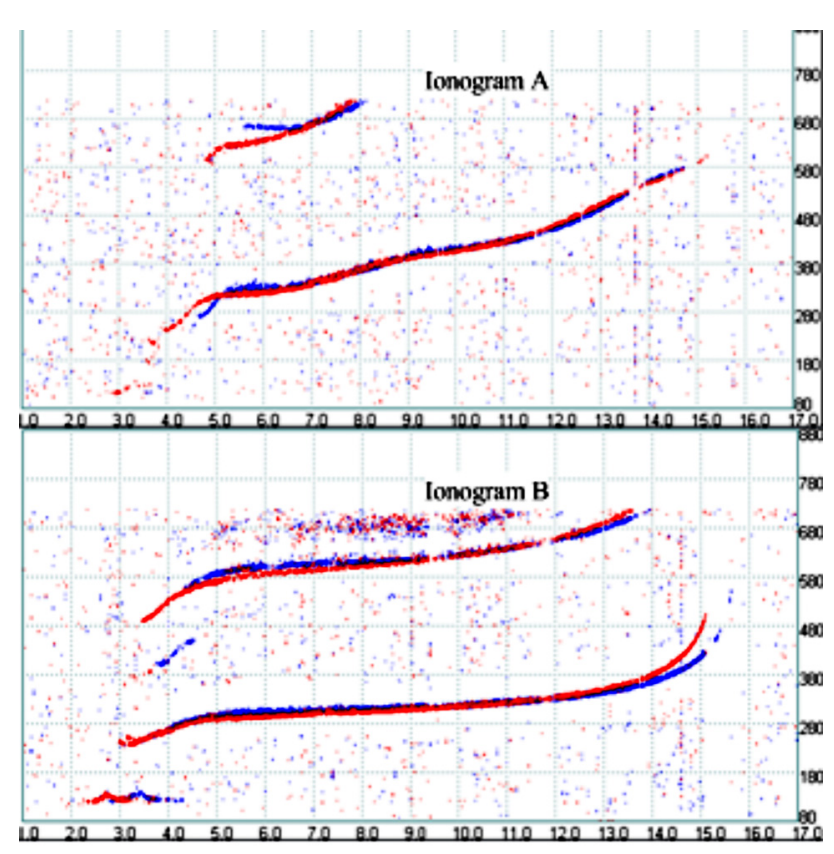

Figure 2. The selected background ionograms (the critical frequencies of ionograms A and B are 16.6 MHzand 15.2 MHz, respectively; in order to derive an entire electron density profiles of O-mode and X-mode from ionogram A, extend the ionogram traces reasonably using extrapolation). 


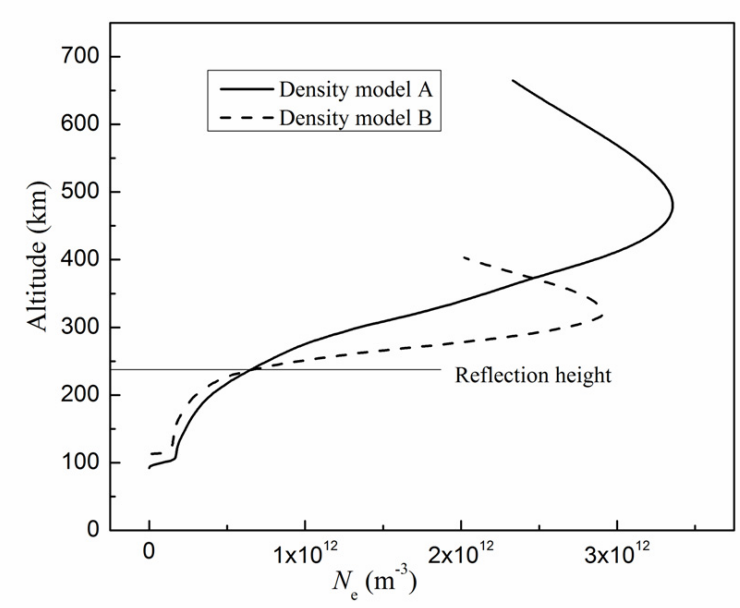

Figure 3. The selected background electron density profiles deduced from two measured ionograms (Figure 2).

Chen 1974], which is inversely proportional to $\nabla N$

$$
H=\left[\frac{f_{p}^{2}}{f^{2}} \frac{\partial N}{\partial Z}\right]^{-1}
$$

The electric field of O-mode near the reflection altitude behaves as a standing wave and undergoes an amplitude swelling. The swelling factor of the incident power density just below the reflection height [Kantor $1972]$ is

$$
A=7.2\left[\frac{\omega H}{c}\right]^{1 / 3}
$$

The standing wave pattern near the reflection height has a significant relationship with the possible excitation of parametric processes and thus the HF wave energy absorption. Under certain conditions, anomalous absorption will play an important role. A smaller electron density gradient, which means a larger swelling factor, will lead to a larger heating effect.
2.2.3. Influences of the ratio of $f_{\mathrm{HF}}$ to foF 2 to on heating effects

The background ionosphere is controlled by solar activity and its critical frequencies vary in a large range in a day, especially during high solar activity. As a comparison, the heating parameters can remain constant in certain periods due to limits of heating conditions or experimental setup. In order to investigate the influence of the ratio of $f_{\mathrm{HF}}$ to $f_{\mathrm{oF}} 2$ on heating effects and compare results under different conditions, we do the simulations as follows: (1) assume $f_{\mathrm{HF}}$ as a constant and set foF 2 different values in a day; (2) keep foF 2 as a constant and change $f_{\mathrm{HF}}$. Here the heating effects at Guangzhou with $P_{\text {erf }}=200 \mathrm{MW}$ are simulated. $f_{\mathrm{HF}}$ is equal to $6.5 \mathrm{MHz}$ and foF2 ranges from $7 \mathrm{MHz}$ to 15.5 $\mathrm{MHz}$ in the first case while foF2 remains fixed at 15.2 $\mathrm{MHz}$ and $f_{\mathrm{HF}}$ ranges from $6 \mathrm{MHz}$ to $15 \mathrm{MHz}$ in the second case. The results are plotted in Figure 5 and Figure 6 respectively.

The electron density depletion $N_{1} / N_{0}$ and temperature increment $T_{\mathrm{e} 1} / T_{\mathrm{e} 0}$ has a close relationship with the ratio of $f_{\mathrm{HF}}$ to $f \mathrm{OF} 2$ (see Figure 5). The critical frequency foF 2 is inclined to change quickly in a day but the HF frequency $f_{\mathrm{HF}}$ remains constant and smaller than $f_{\mathrm{oF}} 2$ all the time. The heating effects are getting better as $f_{\mathrm{HF}} / f_{\mathrm{oF}} 2$ approaches to 1 . As foF2 increases (hence the ratio $f_{\mathrm{HF}} / f_{\mathrm{OF}} 2$ decreases), the reflection height $h_{0}$ firstly shows a drop until $f_{\mathrm{HF}} / f \mathrm{fO} 2=0.65$ and then shows a rise. They influence heating effects together, resulting in an appreciable density disturbance under the condition of low $f_{\mathrm{HF}} / f_{\mathrm{OF}} 2$, as shown by the local maximum at $f_{\mathrm{HF}} / f_{\mathrm{OF}} 2 \approx 0.65$. These findings are quite helpful for the selection of $f_{\mathrm{HF}}$.

A similar result can be found more clearly in Figure 6 , where a maximum of $N_{1} / N_{0}$ occurs when

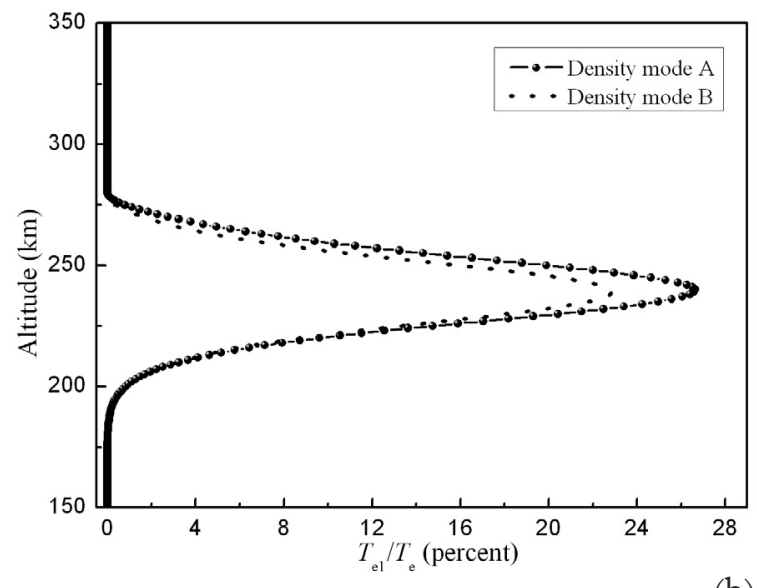

(b)

Figure 4. Relative variation profiles of electron density (left) and electron temperature (right) caused by heating with different background density gradients. 


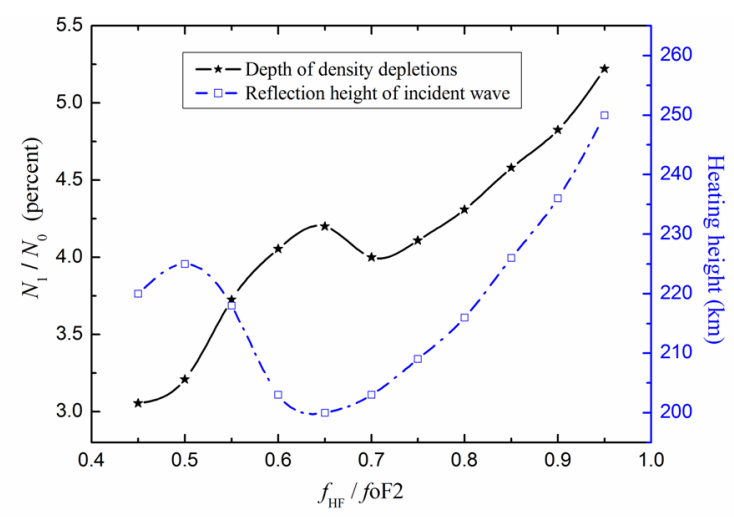

(a) Density depletion

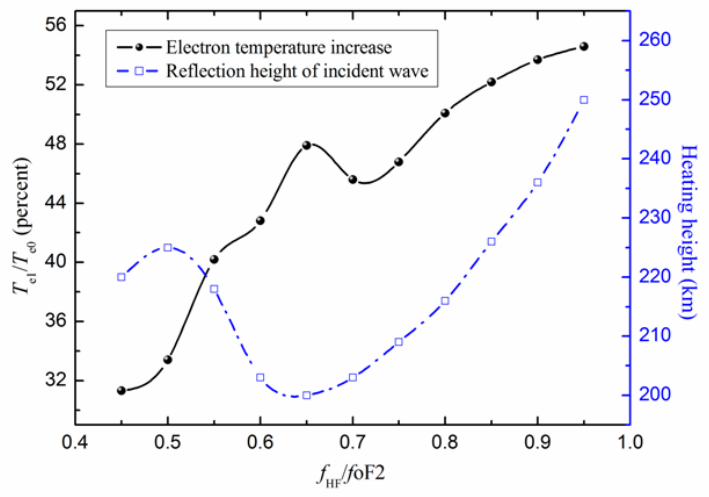

(b) Temperature increment

Figure 5. Influences of the ratio of $f_{\mathrm{HF}}$ to $f \circ \mathrm{OF} 2$ on heating effects with a constant $f_{\mathrm{HF}}$ and different values of $f_{\mathrm{oF}}$. The solid line with asterisks represents the density depletion $N_{1} / N_{0}$ (left) and temperature increment $T_{\mathrm{e} 1} / T_{\mathrm{e} 0}$ (right), the broken line with squares represents $h_{0}$.

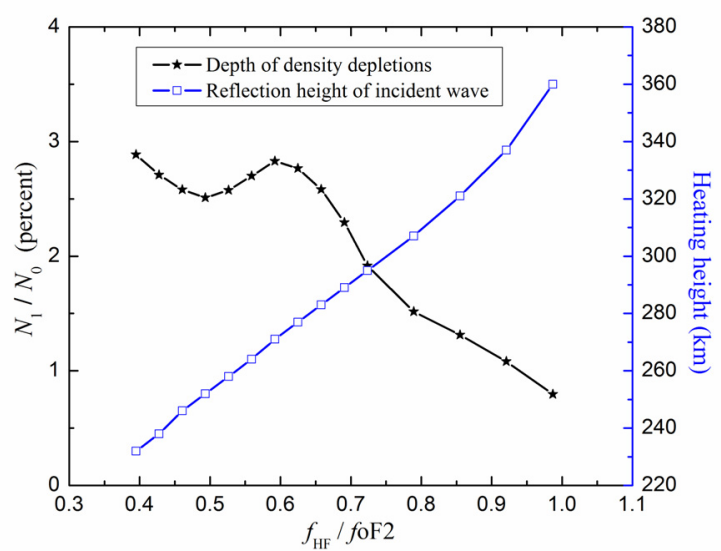

(a) Density depletion

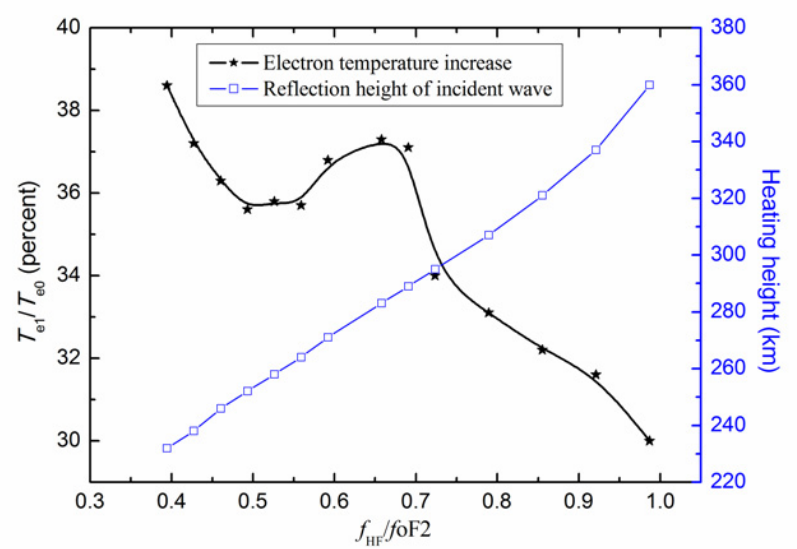

(b) Temperature increment

Figure 6. Same as Figure 5. but for a constant $f \circ \mathrm{F} 2(=15.2 \mathrm{MHz})$ and different values of $f_{\mathrm{HF}}$.

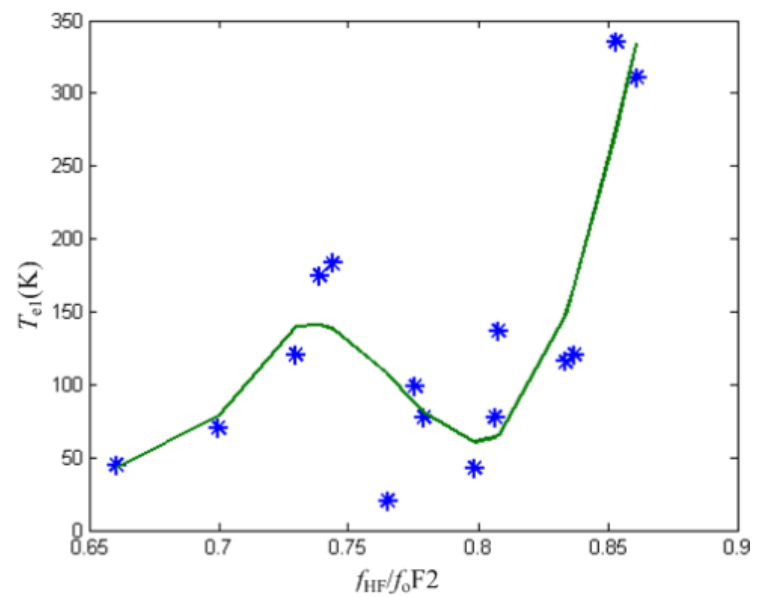

(a) Temperature increment

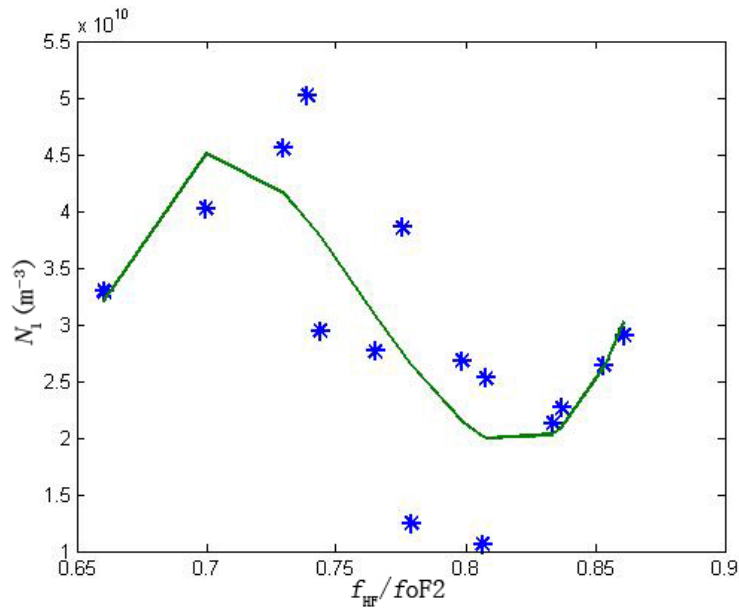

(b) Density depletion

Figure 7. Relative variation profiles of electron temperature and density caused by heating with different values of ratio $f_{\mathrm{HF}} / f_{\mathrm{O}} \mathrm{F} 2$. The observations are indicated with asterisks and the fitted curve with solid line.

$f_{\mathrm{HF}} / f_{\mathrm{oF}} 2$ ranges form 0.5 to 0.7 . When $f \mathrm{oF} 2$ is constant, the reflection height and $f_{\mathrm{HF}} / f \mathrm{OF} 2$ varies in phase with $f_{\mathrm{HF}}$. Their increases have a positive and a negative impact on heating effects, respectively. Unlike the first case shown in Figure 5, the reflection height shown in Figure 6 covers a larger range and thus has a more effective impact on variations of electron density. With $f_{\mathrm{HF}} / f_{\mathrm{O}} \mathrm{F} 2$ less than 0.5 , the reflection height plays a dominant role and the increase of $f_{\mathrm{HF}}$ leads to a decrease of $N_{1} / N_{0}$. As $f_{\mathrm{HF}}$ increases up to a certain value $f_{\mathrm{HF}} / f_{\mathrm{O}} \mathrm{F} 2$ becomes dominant and $N_{1} / N_{0}$ begins increasing. With a further increase of $f_{\mathrm{HF}} / f_{\mathrm{oF}} 2$ larger than 0.7 , the reflection 
height shows its dominance on heating effects again. The temperature increment shows a similar trend.

The results imply that there might be a better ratio range with small values of the ratio of $f_{\mathrm{HF}}$ to $f_{\mathrm{oF}} 2$, which is supported further by an experimental example. I.e., the experiment study of influences of the ratio of $f_{\mathrm{HF}}$ to $f_{0} \mathrm{~F} 2$ on heating effects was conducted using the EISCAT facility on August 17, 2009. The facility radiated O-mode waves at the frequency $f_{\mathrm{HF}}=4.04 \mathrm{MHz}$ with $P_{\text {erf }}=120 \mathrm{MW}$ in the following mode: 8-min radiation 4-min pause in the experiment. The results (the electron temperature) were dealt with using average value of each period, which are shown in Figure 7. When the value $f_{\mathrm{HF}} / f_{\mathrm{OF}} 2$ is small, the variation of the electron temperature is small, when $f_{\mathrm{HF}}$ is close to $f \circ \mathrm{F} 2$, the variation of the electron temperature is relatively large, but meanwhile there is a peak at $f_{\mathrm{HF}} / f_{\mathrm{OF}} 2=0.74$. The electron density perturbation shows a similar trend.The experimental result shows that there was a better ratio range with a small $f_{\mathrm{HF}} / f_{\mathrm{oF}} 2$, which can be used during the ionospheric heating to obtain relatively effective results.

\section{Conclusions}

In this paper, we have simulated the heating effects in the low- and high-latitude under the equivalent conditions stemming from the well-known ionospheric heating experiments at Arecibo as well as at Tromsø, respectively. The findings have been extensively exploited to verify the validation of our model by comparison to the experimental results. Further, the analysis has been carried out on the influences of the dip angle, the background electron density gradient and the ratio $f_{\mathrm{HF}} / f_{\mathrm{O}} \mathrm{F} 2$ on heating effects. Finally we come to draw the following conclusions:

(1) Under certain conditions, a smaller electron density gradient of background ionospheric $\mathrm{F}$ region leads to a better ionospheric heating effect. When the background electron density suffers a rapid change, the altered gradient affects heating effects.

(2) During over-dense heating, the heating effects are enhanced if the ratio of $f_{\mathrm{HF}}$ to $f \mathrm{oF} 2$ increases, which is slightly limited by the resultant elevation of the reflection height. However, there might be a better ratio range with small values of the ratio $f_{\mathrm{HF}}$ to $f_{\mathrm{OF}} 2$, e.g., $[0.5,0.7]$ in the current study.

According to the simulation results in the paper, several measures can be taken to improve heating effects under unfavorable background conditions. When the height of ionospheric $F$ region rises up, we may increase the $P_{\text {erf }}$ of the heating facilities to increase the incident power density of the heated volume. When the ionospheric critical frequency shifts rapidly, we may make use of a short duty cycle and switch pump frequencies swiftly. If the capacity of the available heating system is limited, we may choose matched times with low ionospheric height, critical frequencies or gradient of electron density to obtain relatively effective results.

We considered the influences of the ratio $f_{\mathrm{HF}} / f_{\mathrm{oF}} 2$ and the reflection height together which have been neglected by the previous theoretical investigations. During the experiments, especially at noon during high solar activity, unfavorable background conditions are often encountered without choice, in which we can adjust heating parameters adaptively.

Acknowledgements. We are grateful to Li Qiang for useful suggestions. The authors are appreciated to the EISCAT Scientific Association. EISCAT is an international association supported by China (CRIRP), Finland (SA), Japan (NIPR and STEL), Norway (NFR), Sweden (VR) and the United Kingdom (NERC). This work is supported by the NSFC with grant No. 41004065 .

\section{References}

Banks, P.M., and G. Kockarts (1972). Aeronomy, part B, Academic Press, NewYork.

Blagoveshchenskaya, N.F., A.D. Andreev et al. (1995). Ionospheric wave processes during HF heating experiments, Adv. Space Res, 15 (12), 45-48.

Blagoveshchenskaya, N., F. Borisova et al. (2011). The effects of modification of a high-latitude ionosphere by high-power HF radio waves. Part 1. Results of multi-instrument ground-based observations, Radiophysics and Quantum Electronics, 53 (9), 512-531.

Das, A.C., and J.A. Fejer (1979). Resonance Instability of Small-Scale Field-Aligned Irregularities, J. Geophys. Res, 84 (A11), 6701-6704.

Duncan, L.M., and J.P. Sheerin (1985). High-Resolution Studies of the HF Ionospheric Modification Interaction Region, J. Geophys. Res, 90 (A9), 8371-8376.

Duncan, L.M., J.P. Sheerin et al. (1988). Observations of Ionospheric Cavities Generated by High-Power Radio Waves, Phys. Rev. Lett, 61 (2), 239-242.

Frolov, V., L. Kagan et al. (1999). Review of features of stimulated electromagnetic emission (see): Recent results obtained at the SURA heating facility, Radiophysics and Quantum Electronics, 42 (7), 557-561.

Gordon, W.E., R. Showen et al. (1971). Ionospheric Heating at Arecibo: First Tests, J. Geophys. Res., 76 (31), 7808-7813.

Gurevich, A V. (1978). Nonlinear Phenomena in the Ionosphere, Springer, Berlin.

Gustavsson, B., M.T. Rietveld et al. (2010). Rise and fall of electron temperatures: Ohmic heating of ionospheric electrons from underdense HF radio wave pumping, J. Geophys. Res, 115 (A12), A12332.

Hansen, J.D. (1990). Large-scale ionospheric modifca- 
tion by high-power radio waves: theory and observation, $\mathrm{PhD}$, University of California, Los Angeles. Hansen, J.D., G.J. Morales et al. (1992). Large-Scale HFInduced Ionospheric Modifications: Theory and Modeling, J. Geophys. Res, 97 (A11), 17019-17032.

Hinkel, D., M. Shoucri et al. (1992). Modeling of HF propagation and heating in the ionosphere, Final Technical Report, TRW space and technology group.

Isham, B., W. Birkmayer et al. (1987). Observations of Small-Scale Plasma Density Depletions in Arecibo HF Heating Experiments, J. Geophys. Res, 92 (A5), 4629-4637.

Kantor, I.J. (1972). Plasma waves induced by HF radio waves, $\mathrm{PhD}$, Rice University.

Kosch, M.J., T. Pedersen et al. (2007). Artificial optical emissions in the high-latitude thermosphere induced by powerful radio waves: An observational review, Adv. Space Res, 40 (3), 365-376.

Mantas, G.P., H.C. Carlson Jr. et al. (1981). Thermal Response of the $\mathrm{F}$ Region Ionosphere in Artificial Modification Experiments by HF Radio Waves, J. Geophys. Res, 86 (A2), 561-574.

Meltz, G., L.H. Holway Jr. et al. (1974). Ionospheric heating by powerful radio waves, Radio Science, 9 (11), 1049-1063.

Mingaleva, G.I., and V.S. Mingalev (1997). Response of the convecting high-latitude $\mathrm{F}$ layer to a powerful HF wave, Ann. Geophysicae, 15 (10), 1291-1300.

Mingaleva, G.I., V.S. Mingalev et al. (2008). Model Prediction of the Most Effective Frequency for the Large-Scale Modification of the Midlatitude Ionospheric F2 Layer by Powerful HF Radiowaves, Geomagn. Aéron, 48 (1), 66-74.

Pavlov, A.V. (1998). New electron energy transfer rates for vibrational excitation of $\mathrm{N}_{2}$, Ann. Geophysicae, 16 (2), 176-182.

Pavlov, A.V., and K.A. Berrington (1999). Cooling rate of thermal electrons by electron impact excitation of fine structure levels of atomic oxygen, Ann. Geophysicae, 17 (7), 919-924.

Pedersen, T.R., and H.C. Carlson (2001). First observations of HF heater-produced airglow at the High Frequency Active Auroral Research Program facility: Thermal excitation and spatial structuring, Radio Science, 36 (5), 1013-1026.

Rietveld, M.T., B. Isham et al. (2002). HF-Pumpinduced parametric instabilities in the auroral E-region, Adv. Space Res, 29 (9), 1363-1368.

Rietveld, M.T., M.J. Kosch et al. (2003). Ionospheric electron heating, optical emissions, and striations induced by powerful HF radio waves at high latitudes: Aspect angle dependence, J. Geophys. Res, 108 (A4), 1141
Rishbeth, H., and O.K. Garriott (1969). Introduction to ionospheric physics, Academic Press, New York.

Robinson, T.R., A. Stocker et al. (1998). First CUTLASSEISCAT heating results, Adv. Space Res. 21 (5), 663666.

Shoucri, M.M., G.J. Morales et al. (1984). Ohmic Heating of the Polar F Region by HF Pulses, J. Geophys. Res., 89 (A5), 2907-2917.

Tereshchenko, E.D., B.Z. Khudukon et al. (2006). The relationship between small-scale and large-scale ionospheric electron density irregularities generated by powerful HF electromagnetic waves at high latitudes, Ann. Geophysicae, 24 (11), 2901-2909.

Utlaut, W.F. (1970). An Ionospheric Modification Experiment Using Very High Power, High Frequency Transmission, J. Geophys. Res, 75 (31), 6402-6405.

White, P.B., and F.F. Chen (1974). Amplification and absoption of electromagnetic waves in overdense plasmas, Plasma Physics, 16, 565-587.

Wright, D.M., R.S. Dhillon et al. (2009). Excitation thresholds of field-aligned irregularities and associated ionospheric hysteresis at very high latitudes observed using SPEAR-induced HF radar backscatter, Ann. Geophysicae, 27 (7), 2623-2631.

Xu Bin, Wu Jun, Wu Jian and Wu Zhensen et al. (2009). Observations of the heating experiments in polar winter ionosphere, Chinese J. Geophys., 52 (2), 322341 (in English).

${ }^{\star}$ Corresponding author: Wu Zhensen,

Xidian University, Department of Physics, Xi'an, China; email:wuzhs@mail.xidian.edu.cn.

C 2013 by the Istituto Nazionale di Geofisica e Vulcanologia. All rights reserved. 


\section{Appendix A: Theoretical model}

According to the basic theory of ionospheric heating, microscopic modification of the electron temperature and density in the $\mathrm{F}$ region can be modeled by fluid equations [Meltz et al. 1974, Gurevich 1978]. The three physical equations involved in our simulation model are the momentum equation, the continuity equation and the energy equation.

\section{A.1. The momentum equation}

Considering four species of singly charged ions and electron $\left(\mathrm{O}^{+}, \mathrm{NO}^{+}, \mathrm{O}_{2}^{+}, e\right)$ and neglecting the inertia term, the momentum equation can be written as follows:

$$
\begin{aligned}
& -\nabla p_{\alpha}+N_{\alpha} q_{\alpha} \boldsymbol{E}+q_{\alpha} \mathbf{v}_{\alpha} \times \boldsymbol{B}-N_{\alpha} m_{\alpha} \boldsymbol{g}- \\
& -N_{\alpha} m_{\alpha}\left(\mathbf{v}_{\alpha}-\mathbf{v}_{\mathbf{n}}\right) \boldsymbol{v}_{\alpha \mathbf{n}}-N_{\alpha} m_{\alpha} \sum_{\beta \neq \alpha}\left(\mathbf{v}_{\alpha}-\mathbf{v}_{\beta}\right) \boldsymbol{v}_{\alpha \beta}=0
\end{aligned}
$$

where the subscripts $\alpha, \beta$ refer to the different types of charged particles $\left(\mathrm{O}^{+}, \mathrm{NO}^{+}, \mathrm{O}_{2}^{+}, e\right), n$ the neutral particles. $p_{\alpha}=n_{\alpha} k_{\mathrm{b}} T_{\alpha}$ is the pressure of species $\alpha$, and $N_{\alpha}$, $m_{\alpha}, T_{\alpha}, \mathbf{v}_{\alpha}$ are density, mass, temperature and drift velocity, respectively. $g$ is the acceleration of gravity and $k_{\mathrm{b}}$ is Boltzmann's constant. The collisional rate $v_{\alpha \mathrm{n}}$ and $\nu_{\alpha \beta}$ represent collisions of species $\alpha$ with neutral particles and species $\beta$, respectively. Summing Equation (A1) over the charged species, the collisional terms among charged particles are deleted completely, and the electromagnetic force terms vanish with the hypotheses of quasi-neutrality and ambipolar diffusion. Introducing a mass-weighted ionic collisional term $M_{\mathrm{I}} v_{\text {In }}$ [Hinkel et al. 1992] and the diffusion coefficient $D$

$$
\frac{1}{M_{\mathrm{I}} v_{\text {In }}}=\sum_{\mathrm{i}} \frac{\left(n_{\mathrm{i}} / n_{\mathrm{e}}\right)}{m_{\mathrm{i}} v_{\text {in }}}, D=\frac{1}{m_{\mathrm{e}} v_{\text {en }}+M_{\mathrm{I}} v_{\text {In }}}
$$

and assuming the three species ions have the identical temperature $T_{\mathrm{i}}$ then the momentum equation in the direction of the geomagnetic field can be written as

$$
\begin{aligned}
& N_{e} \mathbf{v}_{e}= \\
& -D\left[\nabla N_{\mathrm{e}} k_{\mathrm{b}}\left(T_{\mathrm{e}}+T_{\mathrm{i}}\right)+\sum_{\alpha} N_{\alpha} m_{\alpha} g\right]+N_{\mathrm{e}} \mathbf{v}_{\mathrm{n}} \cos I,
\end{aligned}
$$

where $v_{\text {en }}$ and $v_{\text {In }}$ are collisional frequencies of electron and ions with neutral particles, respectively, and $I$ is the geomagnetic dip.

\section{A.2. The continuity equation}

The main ion constituent in the F-region is atomic $\mathrm{O}^{+}$, which does not recombine with electrons directly [Rishbeth and Garriot 1969]. Instead, $\mathrm{O}^{+}$undergoes an ion-atom interchange reaction with neutral particles (mainly $\mathrm{N}_{2}$ and $\mathrm{O}_{2}$ ) and the resultant molecular ions com- bine with electrons, known as the $\beta$-type recombination, which has no dependence on electron temperature

$$
\begin{aligned}
& \mathrm{O}^{+}+\mathrm{N}_{2} \rightarrow \mathrm{NO}^{+}+\mathrm{N}, \mathrm{O}^{+}+\mathrm{O}_{2} \rightarrow \mathrm{O}_{2}^{+}+\mathrm{O} \\
& \mathrm{NO}^{+}+e^{-} \rightarrow \mathrm{N}+\mathrm{O}, \mathrm{O}_{2}^{+}+e^{-} \rightarrow \mathrm{O}+\mathrm{O}
\end{aligned}
$$

When ignoring the density variations of $\mathrm{N}_{2}, \mathrm{O}_{2}$ and $\mathrm{O}^{+}$during the heating process, the enhancement of electron temperature has little effect on the production of $\mathrm{NO}^{+}$and $\mathrm{O}_{2}^{+}$. Therefore, the continuity equation takes the form:

$$
\frac{\partial N_{e}}{\partial t}=Q_{0}-\left(k_{1} N_{\mathrm{NO}^{+}}+k_{2} N_{\mathrm{O}_{2}^{+}}\right) N_{\mathrm{e}}-\nabla \cdot\left(N_{\mathrm{e}} \mathbf{v}_{\mathrm{e}}\right),
$$

where $k_{1}=4.2 \times 10^{-7}\left(300 / T_{\mathrm{e}}\right)^{0.85} \mathrm{~cm}^{3} / \mathrm{s}$ and $k_{2}=$ $1.6 \times 10^{-7}\left(300 / T_{\mathrm{e}}\right)^{0.55} \mathrm{~cm}^{3} / \mathrm{s}$ are dissociative recombination coefficients, $Q_{0}$ is electron production rate in the equilibrium state $(\partial / \partial t=0)$ without external electric fields.

\section{A.3. The energy equation}

The energy equation can be written as follows:

$$
\begin{gathered}
\frac{3}{2} k_{\mathrm{b}}\left[N_{\mathrm{e}} \frac{\partial T_{\mathrm{e}}}{\partial t}+N_{\mathrm{e}} \mathbf{v}_{\mathrm{e}} \nabla T_{\mathrm{e}}\right]+k_{\mathrm{b}} N_{\mathrm{e}} T_{\mathrm{e}} \nabla \mathbf{v}_{\mathrm{e}}= \\
\nabla\left(K_{\mathrm{e}} \nabla T_{\mathrm{e}}\right)+S_{\mathrm{HF}}+S_{0}-L .
\end{gathered}
$$

Equation (A6) is a time-dependent expression for electron temperature including the effects of convection, compression, conduction, heat production and loss. The left terms are the change rate of electron energy, the convection, and compression rate. The first term on the right side is the heat conduction rate. The heat conduction across the magnetic field can be ignored and the parallel thermal conductivity $K_{\mathrm{e}}$ [Banks and Kockarts 1972] is

$K_{\mathrm{e}}=$

$\frac{7.7 \times 10^{5} T_{\mathrm{e}}^{5 / 2}}{1+3.22 \times 10^{4}\left(T_{\mathrm{e}}^{2} / N_{\mathrm{e}}\right) \sum_{\mathrm{n}} N_{\mathrm{n}} \bar{Q}_{D}} \mathrm{eV} \cdot \mathrm{cm}^{-1} \cdot \mathrm{s}^{-1} \cdot \mathrm{K}^{-1}$

where, $N_{\mathrm{n}}$ represents the densities of neutral particles and $\bar{Q}_{\mathrm{v}}$ the transfer cross-section of mean momentum for corresponding neutral particles [Banks and Kockarts 1972]. A complete theoretical description of the absorption of the HF wave $S_{\mathrm{HF}}$ involves ohmic heating due to deviative absorption, linear mode conversion to electrostatic waves, parametric processes and Langmuir collapse, and the slow-down of super thermal electrons accelerated by the mode-converted waves. The exact amount of energy deposited in the ionospheric electrons in this region is therefore difficult to calculate accurately from first principles. Here following the simple absorption model proposed by Hansen [Hansen 1990], we uti- 
lize a Gaussian profile placed near the reflection layer.

$S_{0}$ and $L$ are the electron heating rate from other sources (mainly the solar radiation) and electron cooling rate, respectively. It is widely accepted that the dominant electron cooling processes involve elastic collisions between electrons and neutrals, rotational and vibrational excitation of molecular species, fine structure transitions in atomic oxygen, and collisions between electrons and ions. The updated estimates of inelastic cross sections associated with cooling rate calculations can be found [Pavlov 1998, Pavlov and Berrington 1999, Gustavsson et al. 2010].

Electron and ion temperatures in the background ionosphere are specified with the IRI-2012 model, and the atmosphere density and temperature are derived from the MSIS-90 model. In our calculations the time step is 0.01 second and the space step is $1 / \sin I \mathrm{~km}$. The simulation processes are as follows: solve Equation (A3) to calculate $\mathbf{v}_{\mathbf{e}}$ according to the background parameters $\left(T_{\mathrm{e} 0}, N_{\mathrm{e} 0}\right.$, etc.); calculate variations of electron temperature $T_{\mathrm{e} 1}$ in the Equation (A6) and then update $T_{\mathrm{e} 0}$ and the recombination and loss rates; obtain variations of electron density $N_{\mathrm{e} 1}$ with Equation (A5), then update $N_{\mathrm{e} 0}$ and the corresponding terms; finish the current calculation loop and step into the next loop until the end of heating. The subscript $e$ in electron density will be omitted for convenience. 